\title{
Towards Accurate Analysis of Particle Size Distribution for Non-Spherically Shaped Nanoparticles as Quality Control Materials
}

\author{
Ulrich Mansfeld $^{1}$, Francesco Pellegrino ${ }^{2}$, Valter Maurino ${ }^{2}$, Sylvie Marguet ${ }^{3}$, Fabienne Testard $^{3}$, Olivier \\ Tache $^{3}$ and Vasile-Dan Hodoroaba ${ }^{1 *}$ \\ 1. Federal Institute for Materials Research and Testing (BAM), Division 6.1 Surface Analysis and \\ Interfacial Chemistry, 12200 Berlin, Germany. \\ 2. Dipartimento di Chimica and Centro Interdipartimentale NIS, Università di Torino, Via Giuria 7, \\ 10125 Turin, Italy. \\ 3. Nimbe, Commissariat à l'énergie atomique et aux énergies alternatives (CEA), CNRS, F-91191 Gif \\ Sur Yvette, France. \\ * Corresponding author: dan.hodoroaba@bam.de
}

Measurement of nanoparticle (NP) size (distribution) becomes a challenging analytical problem when non-spherical NPs must be accurately measured [1]. Most industrial NPs have not only a non-spherical shape but possess a broader (non-monodisperse) size distribution, and due to their agglomeration/aggregation state are difficult (or even impossible) to be addressed individually. Moreover, driven by regulatory purposes related to the identification of a material as a nanomaterial, the accurate measurement of the smallest dimension of a (nano)particulate material makes the analysis even more complex.

Standardized procedures which are able to reliably characterize manufactured nanomaterials are being developed within the European research project nPSize - Improved traceability chain of nanoparticle size measurements. The aim is to develop measurement procedures, reference materials and modelling to improve the traceability chain, comparability and compatibility for NP size measurements to support standardization. Particular attention is paid to non-spherical NPs. Thus, new model nanoparticulate materials with well-controlled shape have been synthesized and supposed to systematic characterization.

A limited number of NP sizing techniques has been selected for their ability to provide traceable dimensional results: microscopy techniques based on a direct observation of NPs: scanning electron microscopy (SEM), transmission electron microscopy (TEM), scanning transmission electron microscopy in transmission mode (STEM-in-TEM), atomic force microscopy (AFM), as well as one ensemble technique small angle X-ray scattering (SAXS).

In the first phase of the project, the efforts are focused on synthesis of nanoparticles of well-defined, non-spherical shape. Following candidates of reference materials (CRM) with certifiable particle size (distribution) are under characterization with respect to their homogeneity and stability: (i) titania nanoplatelets (10-15 nm thickness x 50-60 nm lateral) [2], (ii) titania bipyramides ( 60 nm length x 40 $\mathrm{nm}$ width) [3], (iii) titania acicular particles (100 nm length $\mathrm{x} 15-20 \mathrm{~nm}$ width; aspect ratio 5.5/6), (iv) gold nanorods ( $10 \mathrm{~nm}$ width x $30 \mathrm{~nm}$ length) [4], and (v) gold nanocubes ( $55 \mathrm{~nm}$ x $55 \mathrm{~nm} \times 55 \mathrm{~nm})$ $[5,6]$.

Figure 1 shows representative SEM and STEM-in-SEM images of selected shape-controlled NP materials under investigation. Parallel to the accurate characterization of newly developed reference 
materials candidates, sample preparation procedures as well as measurement and (image) analysis procedures are being also developed in order to enable the accurate analysis of the non-spherically shaped nanoparticles. Further, appropriate measurands and descriptors are defined and evaluated for each nanoparticle material class and for each measurement method in part.

Physical modelling of the signals in electron microscopy techniques (STEM-in-SEM [6], SEM and AFM) and SAXS is being used as a decisive contribution to underpin the measurement traceability of the size of non-spherical nanoparticles, especially with regard to the accurate thresholding/segmentation of the NPs. The organization of a public database with the first results of raw measurement data, their data reduction and corresponding particle size distribution of the new has been just initiated.

\section{References:}

[1] F Babick et al, J. Nanopart. Res. 18:158 (2016), pp. 1-40.

[2] L Mino et al, ACS Appl. Nano Mater. 1 (2018), p. 53556.

[3] V-D Hodoroaba, K Yamamoto and EA Grulke, Microsc. Microanal. 24 (Suppl 1) (2018), p. 1706.

[4] F Hubert et al, Cryst. Growth Des. 12 (2012), p. 1548.

[5] M Pellarin et al, ACS Nano 10 (2016), p. 11266.

[6] S Mitiche et al, J. Phys. Chem. C 121 (2017), p. 4517.

[7] T Klein et al, Meas. Sci. Tech. 22 (2011), pp. 1-9.

[8] This project has received funding from the EMPIR programme co-financed by the Participating States and from the European Union's Horizon 2020 research and innovation programme.

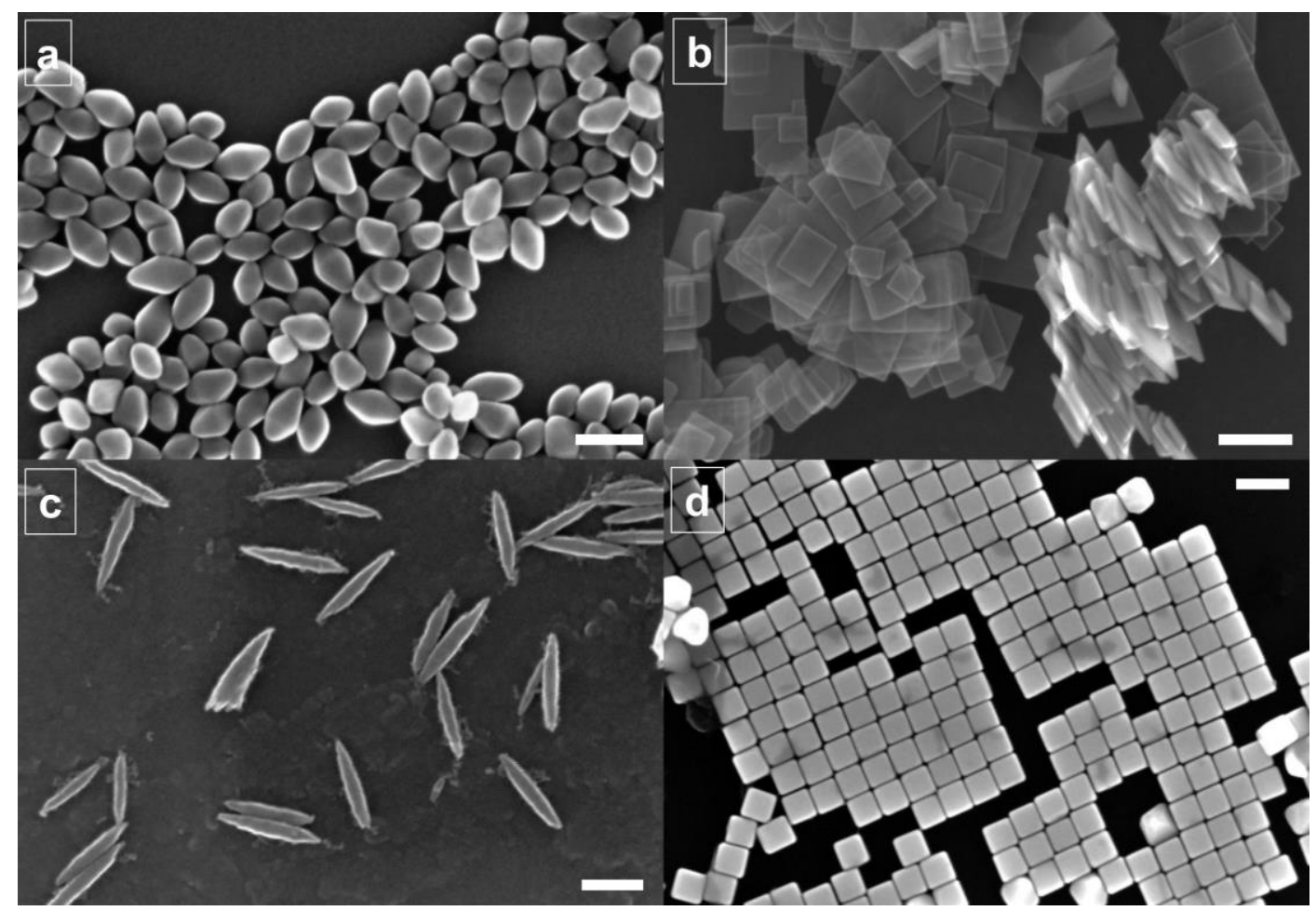

Figure 1. SEM micrographs of the shape-controlled NPs under investigation to be selected as candidate reference materials to be certified for their particle size distribution (a) bipyramidal titania, b) titania nanoplateles, c) acicular titania, and d) Au nanocubes. Scale bar in each image is equal to $100 \mathrm{~nm}$. 